\title{
Electrochromic 2,5-Dihydroxyterephthalic acid linker in metal-organic frameworks
}

\section{Hidetsugu Shiozawa, ${ }^{1,2 *}$ Zuzana Melnikova, ${ }^{2}$ Zdenek Bastl, ${ }^{2}$ Herwig Peterlik, ${ }^{1}$} Martin Kalbac, ${ }^{2}$ Otakar Frank ${ }^{2}$

${ }^{1}$ Faculty of Physics, University of Vienna, Boltzmanngasse 5, 1090 Vienna, Austria

${ }^{2}$ J. Heyrovsky Institute of Physical Chemistry, Czech Academy of Sciences, Dolejskova 3, 182

23 Prague 8, Czech Republic

*To whom correspondence should be addressed; E-mail: hide.shiozawa@jh-inst.cas.cz \& hidetsugu.shiozawa@univie.ac.at

\begin{abstract}
Metal-organic frameworks (MOFs) are diverse in colour owing to a large variety of molecular structures. Here we report electrochromism of M-MOF-74 (M=Mg, Mn, Co or Zn), a honeycomb nano-framework in which hexagonally packed one-dimensional arrays of metal cations are coordinated with 2,5-Dihydroxyterephthalic acid linker. Raman spectroscopy upon electrochemical doping combined with density functional theory calculations reveals redox reactions of the linker while the metal cations stay divalent as probed by X-ray photoemission spectroscopy. Excellent adhesion of the MOFs to glass allows synthesis of quality thin films to be implemented into electrochromic devices that exhibit promising colour contrast and durability.
\end{abstract}

\section{Introduction}

The electrochromic effect, that is material's colour change upon faradaic charge transfer, is known to occur in various inorganic (transition metal-oxides ), organic (molecules [1] and conducting polymers [2, 3, 4]), and organic-inorganic hybrid materials (coordination polymers [5] and metal complexes[6] [7]). Metal oxides are the most studied and they exhibit relatively high durability, but their synthesis requires expensive vacuum deposition systems. Organic or hybrid materials have the advantage of inexpensive production, but suffer from low stability and durability. In metal-oxides and metal complexes, reversible electrical switching of the metal's oxidation state accompanies colour modulations. 
Coordination polymers, known as metal-organic frameworks (MOFs) $[8][9$, are naturally colourful owing to a variety of metal-ligand combinations available. Their open nanometric pores ensure effective doping as every active site can be accessed by electrolyte molecules with minimal structural deformation. To date, several electrochromic MOFs were tailored based on known electrochromic molecules such as naphthalene diimide [10] [11] [12] [13] [14] [15] [16] as linkers that function as chromophores in the framework [17].

Generally, the fact that many MOFs change their colour reversibly upon solvent exchange or sorption of various molecules suggests their potential as electrochromic materials [18] [19] 20] 21] [22] 23] [24], specifically those having open-metal sites that can capture ions [25] [26] [27] or molecules [28] [29] [30].

Electrochromic effects reported on MOFs with non-electrochromic linkers support the importance of the role of metal ions [31]. Two-dimensional coordination nanosheets (CONASH) with iron or cobalt were synthesised to exhibit electrochromic effects that were attributed to the redox reaction of iron or cobalt as in bis(terpyridine)iron or bis(terpyridine)cobalt complex wires [32].

MOF-74, also known as CPO-27, M2(dhtp), or M2(dobdc) where M is divalent Mg, Mn, $\mathrm{Fe}, \mathrm{Co}, \mathrm{Ni}$, or $\mathrm{Zn}$, and dhtp (or dobdc) is tetravalent 2,5-dihydroxyterephthalic acid (2,5dioxido-1,4-benzenedicarboxylate), is a family of MOFs that was extensively studied for gas sorption as the densely packed open metal sites are expected to interact effectively with gaseous molecules [33] [34] 29] [26] [35]. Among them, Zn-MOF-74 was reported to be electrochromic, but no mechanism for this effect was suggested [31]. Co-MOF-74 is reportedly redox-active as an anode material for lithium-ion batteries [36]. Stoichiometric oxidation of Mn-2(2,5-dioxidoterephthalate) (Mn-MOF-74) was demonstrated by chlorination of manganese cations with a mild oxidation agent, iodobenzene dichloride $\left(\mathrm{PhICl}_{2}\right)$ [26], as well as by fluorination and doping with TCNQ [37] 38].

In the present work, we synthesize high quality thin films of M-MOF-74 $(\mathrm{M}=\mathrm{Mg}, \mathrm{Mn}$, Co, or Zn) directly on fluorine-doped tin oxide (FTO)-coated glass plates. We demonstrate that the colour of all these films changes from ochre to brown upon cathodic electrochemical doping. The electrochromism observed universally for this family of MOFs with the least to the most redox-active transition metals suggests that the linker is redox active. Among 
them, Mn-MOF-74 with the redox-active $\mathrm{Mn}^{2+}$ is the best suited material for a deeper study in which the origin of the redox reaction can be identified. In-situ Raman spectroelectrochemistry on Mn-MOF-74 reveals a redox reaction of the dhtp linker as the cause of the electrochromism, that is in line with the divalent Mn state unchanged upon solid-state doping using an ionic liquid as probed by $\mathrm{X}$-ray photoemission spectroscopy. The strong $\mathrm{C}=\mathrm{O}$ stretching mode of the dhtp emerging upon doping is reproduced by density functional theory calculations on a model electron-doped dhtp system. Finally, the scalable synthesis method utilizing excellent adhesion of the MOFs on FTO-coated glass plates allows fabrication of cathodic electrochromic devices with high durability and colour contrast as demonstrated with Mn-MOF-74.

\section{Results and discussion}

Cyclic voltammetry of the M-MOF-74 ( $\mathrm{M}=\mathrm{Mg}$, Mn, Co or Zn) thin film prepared on FTOcoated glass is performed in $0.2 \mathrm{M} \mathrm{LiClO}_{4}$ acetonitrile electrolyte solution in the homemade electrochemical cell, as shown in Fig. 1. It is shown that the colour of M-MOF-74 thin films on the FTO-coated glass turns dark upon electrochemical doping with positive biasing as excess $\mathrm{ClO}_{4}^{-}$anions neutralise the charge of the M-MOF-74 working electrode. The same colour change has also been observed with other electrolyte solutions such as $\mathrm{KCl}$ in water

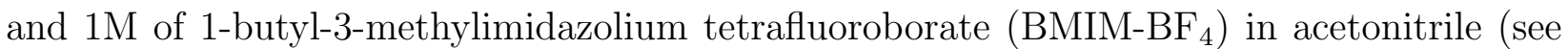
SI). The corresponding cyclic voltammogram at a scan rate of $0.1 \mathrm{Vs}^{-1}$ in the bias range between -0.2 and $1.0 \mathrm{eV}$ exhibits reproducibly broad redox peaks coinciding with the colour change.

Fig. 2 shows that the peak current versus square root of the scan rate follows a linear function, demonstrating that the redox process is purely diffusion-controlled. From the fit to Randles-Sevcik equation we get a diffusion coefficient of $\sim 4.3 \times 10^{-10} \mathrm{~cm}^{2} \mathrm{~s}^{-1}$. The specific capacity estimated from the $0.01 \mathrm{Vs}^{-1}$ data reaches $29 \mathrm{Cg}^{-1}$ or $8.0 \mathrm{mAhg}^{-1}$ corresponding to $0.095 e$ per M2(dhtp) (see SI).

Spectroelectrochemistry is a powerful tool for elucidating the nature of the electrochromism [39]. The optical absorption spectra of the Mn-MOF-74 film upon electrochemical doping 
are plotted in Fig. 22. The colour change can be associated with an optical absorption peak at $490 \mathrm{~nm}$ emerging upon positive biasing. Further information on the charge state of the framework is gained from Raman spectroelectrochemistry. Fig. 3 a shows the Raman spectra of the Mn-MOF-74 film measured at a laser wavelength of $514.5 \mathrm{~nm}$ upon positive biasing in $0.2 \mathrm{M} \mathrm{LiClO}_{4}$ acetonitrile electrolyte solution. The spectra at voltages up to $0.4 \mathrm{~V}$ exhibit multiple spectral features including three prominent peaks at Raman frequencies of $560,1284,1403 \mathrm{~cm}^{-1}$, indicated by the dashed black lines, associated with the $\mathrm{C}-\mathrm{H}$ bending of the benzene ring, $\mathrm{C}=\mathrm{O}$ stretching and $\mathrm{O}-\mathrm{C}-\mathrm{O}$ symmetric stretching modes of the dhtp ${ }^{\delta_{0}-}$ ligand, respectively, where $\delta_{0}$ (tentatively 4 ) denotes the valency of the dhtp in non-doped Mn-MOF-74. Upon biasing at higher voltages, all three peaks diminish and multiple new peaks emerge at frequencies highlighted by the dashed red lines. The most prominent peak located at $1625 \mathrm{~cm}^{-1}$ was previously observed upon oxidation of the Mn-MOF-74 by fluorination as well as by doping with TCNQ, and can be associated with the ring $\mathrm{C}=\mathrm{O}$ stretching mode of the divalent $\mathrm{dhtp}^{2-}$ ligand, meaning that the framework's linker is stoichiometrically doped [38]. This is also in agreement with the previously reported IR stretching mode of carbonyle group observed in chlorinated Mn-MOF-74 [26].

Here, further insight into the charge state of the doped dhtp can be given by density functional theory calculations. Figure 3c compares the Fourier transform Raman spectrum of neutral dhtp ${ }^{0}$ with our density functional theory result. Despite the use of the local density approximation, agreement between experiment and theory is satisfactory for judging the stoichimetric valency. As calculatons of phonon modes of M-MOF-74 whose unit cell contains 164 atoms are not feasible, we model a compact system with a dhtp sandwitched by two tetrafluoroborate $\left(\mathrm{BF}_{4}^{-}\right)$anions (see the optimized structure in SI). The charge state of the dhtp estimated by the Bader analysis is -1.46. Fig $3 \mathrm{~b}$ shows the Raman lines of this $[\mathrm{dhtp}]^{1.46-}\left[2 \mathrm{BF}_{4}\right]^{1.46-}$. The most prominent line located at $1653 \mathrm{~cm}^{-1}$ is inline with the emerging ring $\mathrm{C}=\mathrm{O}$ peak of the dhtp upon electrochemical doping. This supports the trivalent dhtp, i.e. $\delta=2$, as claimed previously in the doped Mn-MOF-74 materials [38, 26].

Identifying the oxidation state of manganese upon electrochemical doping is challenging, but the electrochemically-doped state of the Mn-MOF-74 can also be sustained to some extent by fast drying the Mn-MOF-film immediately after positive biasing in $0.2 \mathrm{M} \mathrm{LiClO}_{4}$ 
acetonitrile electrolyte solution. Figure 4 a shows the Raman spectrum of the Mn-MOF-74 film that was biased at $0.4 \mathrm{~V}$, then taken out and dried in air. As compared with the spectrum of the non-doped Mn-MOF-74 film, the peak emerging at a frequency of $1611 \mathrm{~cm}^{-1}$ indicated by the dashed red line is consistent with the $\mathrm{C}=\mathrm{O}$ stretching mode of the $\mathrm{dhtp}^{\delta-}$ observed upon electrochemical doping in Fig. 3, meaning that the dried films are still dominantly doped with $\mathrm{ClO}^{4-}$ anion.

These doped solid films allow X-ray photoemission spectroscopy (XPS) analysis of the oxidation state of the manganese ion in the framework (see SI for further details). Figure $4 \mathrm{~b}$ show the XP spectra of the Mn 3s and 2p photoelectrons for the doped and non-doped MnMOF-74 films. The differenece in binding energy between the resolved two $3 \mathrm{~s}$ peaks, ${ }^{7} \mathrm{~S}$ and ${ }^{5} \mathrm{~S}$, located at binding energies of approximately 84 and $90 \mathrm{eV}$, respectively, originate from the exchange coupling between the $3 \mathrm{~s}$ core hole and the $3 \mathrm{~d}$ electrons in the photoemission final state. This exchange splitting energy is known to decrease proportionally as the valence number of manganese increases [40, 41]. Despite the difference in doping level, the exchange splitting energy for both samples is approximately $6 \mathrm{eV}$, indicating that the oxidation state of manganese stays divalent, i.e. $\mathrm{Mn}^{2+}$. Fig. $4 \mathrm{~b}$ shows the Mn 2p XPS spectra of both doped and non-doped Mn-MOF-74 exhibiting the Mn 2p $p_{3 / 2}$ major peak located at $641.7 \mathrm{eV}$ and the shake up satellite at $646.3 \mathrm{eV}$, both typical for $\mathrm{Mn}^{2+}$ [42, 41, 43] These XPS results are in line with the previously reported effective magnetic moment corresponding to a half filled $\mathrm{Mn}^{2+}$ state unchanged upon chlorination of Mn-MOF 74 [26, 44].

Here, the combined Raman and XPS analysis has revealed that the redox reaction of the dhtp linker is responsible for the colour change of the framework upon positive electrochemical doping.

Finally, towards optoelectronic applications, a Mn-MOF-74 film synthesised onto a FTOcoated glass is encapsulated in an electrochromic device together with $0.2 \mathrm{M} \mathrm{LiClO}_{4}$ acetonitrile electrolyte solution, see Fig. 5. The colour of the MOF device changes reversibly upon alternating positive and negative biasing, see the pictures of the device being biased at $-3 \mathrm{~V}$ and $5 \mathrm{~V}$ in Fig. 5 as well as a video in the SI. The corresponding chronoamperogram in Fig. 5 exhibits its performance sustained over 100 cycles. The device reaches a charge of ca. 9 $\mathrm{mC}$ upon biasing at $5 \mathrm{~V}$ for 10 seconds and $-8 \mathrm{mC}$ at $-3 \mathrm{~V}$ for 10 seconds, that are reduced to 
$52 \%$ and $53 \%$, respectively, after 100 cycles, yet the electrochromic effect is sustainable for further cycling.

\section{Conclusion}

Electrochemical doping is a powerful technique to alter physical properties of solid states. We have demonstrated that the M-MOF-74 ( $\mathrm{M}=\mathrm{Mg}, \mathrm{Mn}, \mathrm{Co}$, or Zn) thin film synthesised directly on the transparent electrode exhibit electrochromism that originates from the redox reaction of 2,5-Dihydroxyterephthalic acid liker while the metal oxidation state stays divalent. The electrochromic device fabricated based on the Mn-MOF-74 thin film has shown its promising performance and stability. Provided that all densely-packed redox-active sites are principally accessible throughout the nano-framework of a large volume, electrochromic MOFs of a large variety could serve as viable alternatives to some of the common electrochromic materials.

\section{Acknowledgements}

This work was financially supported by the Austrian Science Fund (FWF), project no. P30431-N36 and the Czech Science Foundation (GACR), project no. 19-15217S and 2008633X. The European Regional Development Fund; OP RDE; Project: "Carbon allotropes with rationalized nanointerfaces and nanolinks for environmental and biomedical applications" (No. CZ.02.1.01/0.0/0.0/16_026/0008382) is also acknowledged. We thank M. Bousa, S. Loyer and A. Stangl for technical assistance.

\section{References}

[1] R. J. Mortimer, A. L. Dyer, and J. R. Reynolds. Electrochromic organic and polymeric materials for display applications. Displays, 27(1):2-18, JAN 2006.

[2] B. L. Groenendaal, F. Jonas, D. Freitag, H. Pielartzik, and J. R. Reynolds. Poly(3,4- 
ethylenedioxythiophene) and its derivatives: Past, present, and future. Advanced Materials, 12(7):481-494, APR 42000.

[3] H. W. Heuer, R. Wehrmann, and S. Kirchmeyer. Electrochromic window based on conducting poly (3,4-ethylenedioxythiophene)poly(styrene sulfonate). Advanced Functional Materials, 12(2):89-94, FEB 2002.

[4] S. Kirchmeyer and K. Reuter. Scientific importance, properties and growing applications of poly( 3,4-ethylenedioxythiophene). Journal of Materials Chemistry, 15(21):20772088, 2005.

[5] H. Masayoshi. Electrochromic Organic-Metallic Hybrid Polymers: Fundamentals and Device Applications. Polymer Journal, 41(7):511-520, 2009.

[6] Neta Elool Dov, Sreejith Shankar, Dana Cohen, Tatyana Bendikov, Katya Rechav, Linda J. W. Shimon, Michal Lahav, and Milko E. van der Boom. Electrochromic MetalloOrganic Nanoscale Films: Fabrication, Color Range, and Devices. Journal of the American Chemical Society, 139(33):11471-11481, AUG 232017.

[7] Michal Lahav and Milko E. van der Boom. Polypyridyl Metallo-Organic Assemblies for Electrochromic Applications. Advanced Materials, 30(41, SI), OCT 112018.

[8] O. M. Yaghi, M. O'Keeffe, N. W. Ockwig, H. K. Chae, M. Eddaoudi, and J. Kim. Reticular synthesis and the design of new materials. Nature, 423(6941):705-714, June 2003.

[9] S. Kitagawa, R. Kitaura, and S. Noro. Functional porous coordination polymers. Angewandte Chemie-international Edition, 43(18):2334-2375, 2004.

[10] E. D. Bloch, L. J. Murray, W. L. Queen, S. Chavan, S. N. Maximoff, J. P. Bigi, R. Krishna, V. K. Peterson, F. Grandjean, G. J. Long, B. Smit, S. Bordiga, C. M. Brown, and J. R. Long. Selective binding of o-2 over n-2 in a redox-active metal-organic framework with open iron(ii) coordination sites. Journal of the American Chemical Society, 133(37):14814-14822, September 2011. 
[11] Ran Li, Kerui Li, Gang Wang, Lei Li, Qiangqiang Zhang, Jinhui Yan, Yao Chen, Qinghong Zhang, Chengyi Hou, Yaogang Li, and Hongzhi Wang. Ion-Transport Design for High-Performance Na+-Based Electrochromics. ACS Nano, 12(4):3759-3768, APR 2018.

[12] Casey R. Wade, Minyuan Li, and Mircea Dinca. Facile Deposition of Multicolored Electrochromic Metal-Organic Framework Thin Films. Angewandte Chemie-international Edition, 52(50):13377-13381, DEC 92013.

[13] Chung-Wei Kung, Timothy Chiaan Wang, Joseph E. Mondloch, David Fairen-Jimenez, Daniel M. Gardner, Wojciech Bury, Jordan Matthew Klingsporn, Jonathan C. Barnes, Richard Van Duyne, J. Fraser Stoddart, Michael R. Wasielewski, Omar K. Farha, and Joseph T. Hupp. Metal-Organic Framework Thin Films Composed of Free-Standing Acicular Nanorods Exhibiting Reversible Electrochromism. Chemistry of Materials, 25(24):5012-5017, DEC 242013.

[14] Yi-Xin Xie, Wen-Na Zhao, Guo-Chang Li, Peng-Fei Liu, and Lei Han. A Naphthalenediimide-Based Metal-Organic Framework and Thin Film Exhibiting Photochromic and Electrochromic Properties. Inorganic chemistry, 55(2):549-551, JAN 18 2016.

[15] Khalid AlKaabi, Casey R. Wade, and Mircea Dinca. Transparent-to-Dark Electrochromic Behavior in Naphthalene-Diimide-Based Mesoporous MOF-74 Analogs. Chem, 1(2), AUG 112016.

[16] Daniel P. Tabor, Loic M. Roch, Semion K. Saikin, Christoph Kreisbeck, Dennis Sheberla, Joseph H. Montoya, Shyam Dwaraknath, Muratahan Aykol, Carlos Ortiz, Hermann Tribukait, Carlos Amador-Bedolla, Christoph J. Brabec, Benji Maruyama, Kristin A. Persson, and Alan Aspuru-Guzik. Accelerating the discovery of materials for clean energy in the era of smart automation. Nature Reviews Materials, 3(5):5-20, MAY 2018.

[17] C-H. Noh, S-J. Jeon, and S-U. Son. Electrochromic material and electrochromic device including the same, 2015. 
[18] Yun-Nan Gong and Tong-Bu Lu. Fast detection of oxygen by the naked eye using a stable metal-organic framework containing methyl viologen cations. Chemical Communications, 49(70):7711-7713, 2013.

[19] Christian Chmelik, Alexander Mundstock, Pascal D. C. Dietzel, and Juergen Caro. Idiosyncrasies of Co-2(dhtp): In situ-annealing by methanol. Microporous and Mesoporous Materials, 183:117-123, JAN 12014.

[20] D. P. Yan, Y. Q. Tang, H. Y. Lin, and D. Wang. Tunable two-color luminescence and host-guest energy transfer of fluorescent chromophores encapsulated in metal-organic frameworks. Scientific Reports, 4:4337, March 2014.

[21] M. D. Allendorf, M. E. Foster, F. Leonard, V. Stavila, P. L. Feng, F. P. Doty, K. Leong, E. Y. Ma, S. R. Johnston, and A. A. Talin. Guest-induced emergent properties in metalorganic frameworks. Journal of Physical Chemistry Letters, 6(7):1182-1195, April 2015.

[22] Gift Mehlana and Susan A. Bourne. Unravelling chromism in metal-organic frameworks. CrystEngComm, 19(30):4238-4259, AUG 142017.

[23] Kirandeep, Ahmad Husain, Ajit Kumar Kharwar, Ramesh Kataria, and Girijesh Kumar. Co(II)-based Metal-Organic Frameworks and Their Application in Gas Sorption and Solvatochromism. Crystal Growth \& Design, 19(3):1640-1648, MAR 2019.

[24] Guang Lu and Joseph T. Hupp. Metal-Organic Frameworks as Sensors: A ZIF-8 Based Fabry-Perot Device as a Selective Sensor for Chemical Vapors and Gases. Journal of the American Chemical Society, 132(23):7832+, JUN 162010.

[25] Carl K. Brozek and Mircea Dinca. Ti3+-, V2+/3+-, Cr2+/3+-, Mn2+-, and Fe2+Substituted MOF-5 and Redox Reactivity in Cr- and Fe-MOF-5. Journal of the American Chemical Society, 135(34):12886-12891, AUG 282013.

[26] Anthony F. Cozzolino, Carl K. Brozek, Ryan D. Palmer, Junko Yano, Minyuan Li, and Mircea Dinca. Ligand Redox Non-innocence in the Stoichiometric Oxidation of Mn2(2,5-dioxidoterephthalate) (Mn-MOF-74). Journal of the American Chemical Society, 136(9):3334-3337, MAR 52014. 
[27] Yuri Tulchinsky, Christopher H. Hendon, Kirill A. Lomachenko, Elisa Borfecchia, Brent C. Melot, Matthew R. Hudson, Jacob D. Tarver, Maciej D. Korzynski, Amanda W. Stubbs, Jacob J. Kagan, Carlo Lamberti, Craig M. Brown, and Mircea Dinca. Reversible Capture and Release of Cl-2 and Br-2 with a Redox-Active Metal-Organic Framework. Journal of the American Chemical Society, 139(16):5992-5997, APR 262017.

[28] David Britt, David Tranchemontagne, and Omar M. Yaghi. Metal-organic frameworks with high capacity and selectivity for harmful gases. Proceedings of the National Academy of Sciences of the United States of America, 105(33):11623-11627, AUG 192008.

[29] Dianne J. Xiao, Eric D. Bloch, Jarad A. Mason, Wendy L. Queen, Matthew R. Hudson, Nora Planas, Joshua Borycz, Allison L. Dzubak, Pragya Verma, Kyuho Lee, Francesca Bonino, Valentina Crocella, Junko Yano, Silvia Bordiga, Donald G. Truhlar, Laura Gagliardi, Craig M. Brown, and Jeffrey R. Long. Oxidation of ethane to ethanol by $\mathrm{N} 2 \mathrm{O}$ in a metal-organic framework with coordinatively unsaturated iron(II) sites. Nature Chemistry, 6(7):590-595, JUL 2014.

[30] Ina Strauss, Alexander Mundstock, Marvin Treger, Karsten Lange, Seungtaik Hwang, Christian Chmelik, Pascal Rusch, Nadja C. Bigall, Thomas Pichler, Hidetsugu Shiozawa, and Jürgen Caro. Metal-organic framework co-mof-74-based host-guest composites for resistive gas sensing. ACS Applied Materials \& Interfaces, 11(15):14175-14181, 2019. PMID: 30900448.

[31] Issam Mjejri, Cara M. Doherty, Marta Rubio-Martinez, Glenna L. Drisko, and Aline Rougier. Double-Sided Electrochromic Device Based on Metal-Organic Frameworks. ACS Applied Materials \& Interfaces, 9(46):39930-39934, NOV 222017.

[32] Kenji Takada, Ryota Sakamoto, Shi-Ting Yi, Shunsuke Katagiri, Tetsuya Kambe, and Hiroshi Nishihara. Electrochromic Bis(terpyridine)metal Complex Nanosheets. Journal of the American Chemical Society, 137(14):4681-4689, APR 152015.

[33] N. L. Rosi, J. Kim, M. Eddaoudi, B. L. Chen, M. O’Keeffe, and O. M. Yaghi. Rod packings and metal-organic frameworks constructed from rod-shaped secondary building units. Journal of the American Chemical Society, 127(5):1504-1518, February 2005. 
[34] Hui Wu, Jason M. Simmons, Gadipelli Srinivas, Wei Zhou, and Taner Yildirim. Adsorption Sites and Binding Nature of CO2 in Prototypical Metal-Organic Frameworks: A Combined Neutron Diffraction and First-Principles Study. Journal of Physical Chemistry Letters, 1(13):1946-1951, JUL 12010.

[35] Mali H. Rosnes, Martin Opitz, Matthias Frontzek, Wiebke Lohstroh, Jan Peter Embs, Peter A. Georgiev, and Pascal D. C. Dietzel. Intriguing differences in hydrogen adsorption in CPO-27 materials induced by metal substitution. Journal of Materials Chemistry A, 3(9):4827-4839, 2015.

[36] Yuxing Liao, Chao Li, Xiaobing Lou, Peng Wang, Qi Yang, Ming Shen, and Bingwen Hu. Highly reversible lithium storage in cobalt 2,5-dioxido-1, 4-benzenedicarboxylate metalorganic frameworks boosted by pseudocapacitance. Journal of Colloid and Interface Science, 506:365-372, NOV 152017.

[37] Hidetsugu Shiozawa, Bernhard C. Bayer, Herwig Peterlik, Jannik C. Meyer, Wolfgang Lang, and Thomas Pichler. Doping of metal-organic frameworks towards resistive sensing. Scientific Reports, 7, MAY 262017.

[38] Michal Blaha, Vaclav Vales, Zdenek Bastl, Martin Kalbac, and Hidetsugu Shiozawa. Host-Guest Interactions in Metal-Organic Frameworks Doped with Acceptor Molecules as Revealed by Resonance Raman Spectroscopy. Journal of Physical Chemistry C, 124(44, SI):24245-24250, NOV 52020.

[39] Pavel M. Usov, Chanel F. Leong, Bun Chan, Mikihiro Hayashi, Hiroshi Kitagawa, Joshua J. Sutton, Keith C. Gordon, Idan Hod, Omar K. Farha, Joseph T. Hupp, Matthew Addicoat, Agnieszka Beata Kuc, Thomas Heine, and Deanna M. D'Alessandro. Probing charge transfer characteristics in a donor-acceptor metal-organic framework by Raman spectroelectrochemistry and pressure-dependence studies. Physical Chemistry Chemical Physics, 20(40):25772-25779, OCT 282018.

[40] VR Galakhov, M Demeter, S Bartkowski, M Neumann, NA Ovechkina, EZ Kurmaev, NI Logachevskaya, YM Mukovskii, J Mitchell, and DL Ederer. Mn 3s exchange splitting in mixed-valence manganites. Physical Review B, 65(11), MAR 152002. 
[41] Eugene S. Ilton, Jeffrey E. Post, Peter J. Heaney, Florence T. Ling, and Sebastien N. Kerisit. XPS determination of Mn oxidation states in Mn (hydr)oxides. Applied Surface Science, 366:475-485, MAR 152016.

[42] Mark C. Biesinger, Brad P. Payne, Andrew P. Grosvenor, Leo W. M. Lau, Andrea R. Gerson, and Roger St. C. Smart. Resolving surface chemical states in XPS analysis of first row transition metals, oxides and hydroxides: Cr, Mn, Fe, Co and Ni. Applied Surface Science, 257(7):2717-2730, JAN 152011.

[43] M Oku, K Hirokawa, and S Ikeda. X-ray photoelectron spectroscopy of manganeseoxygen systems. Journal of Electron Spectroscopy and Related Phenomena, 7(6):465-473, 1975.

[44] M Toupin, T Brousse, and D Belanger. Charge storage mechanism of MnO2 electrode used in aqueous electrochemical capacitor. Chemistry of Materials, 16(16):3184-3190, AUG 102004. 

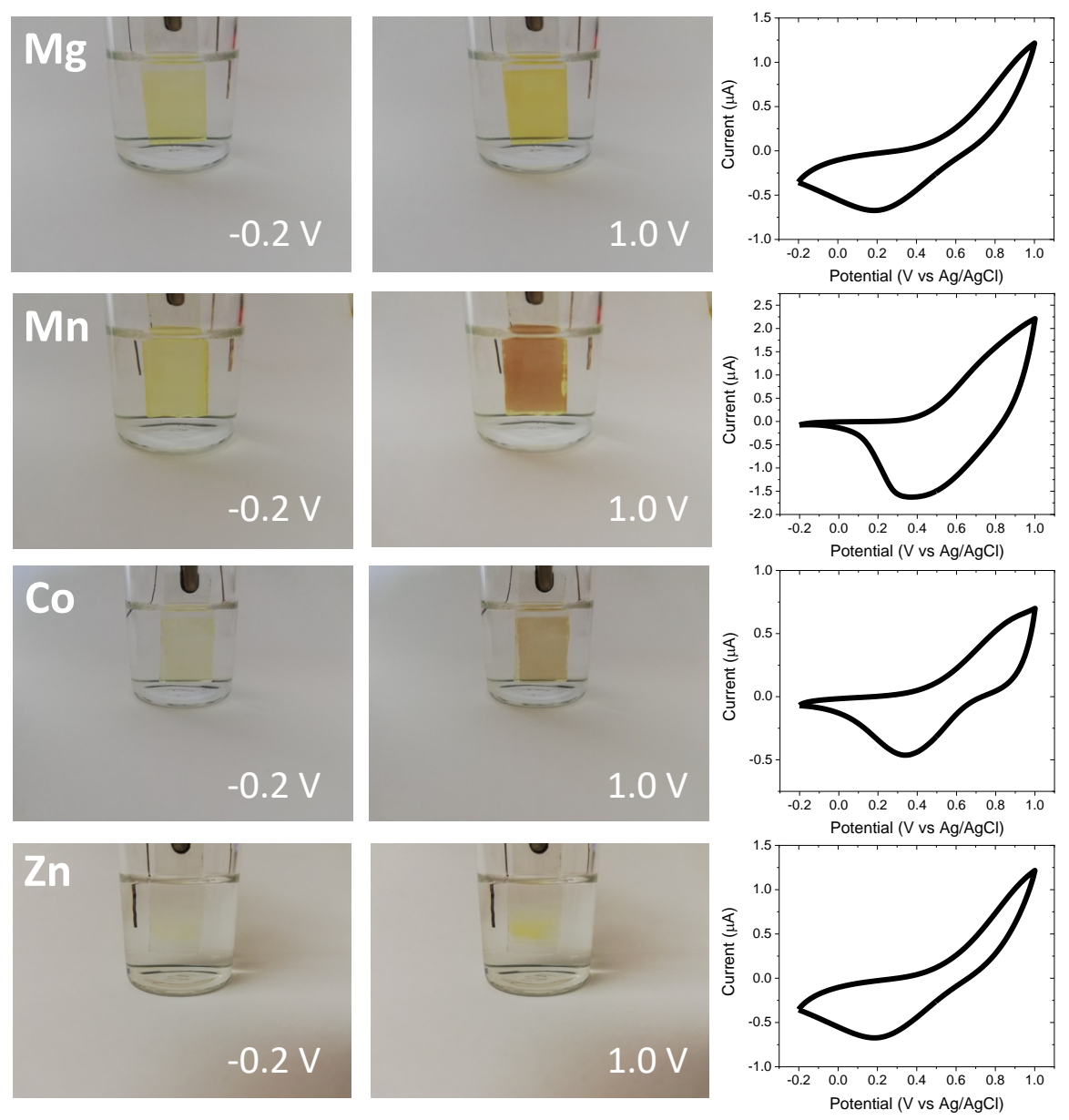

Figure 1: Pictures of thin films of M-MOF-74 $(\mathrm{M}=\mathrm{Mg}, \mathrm{Mn}$, Co and $\mathrm{Zn}$ in the order from the top to the bottom) on FTO-coated glass plates biased at $-0.2 \mathrm{~V}$ (left) and $1.0 \mathrm{~V}$ (center) in $0.2 \mathrm{M} \mathrm{LiClO}_{4}$ acetonitrile electrolyte solution and corresponding cyclic voltammograms (right). 

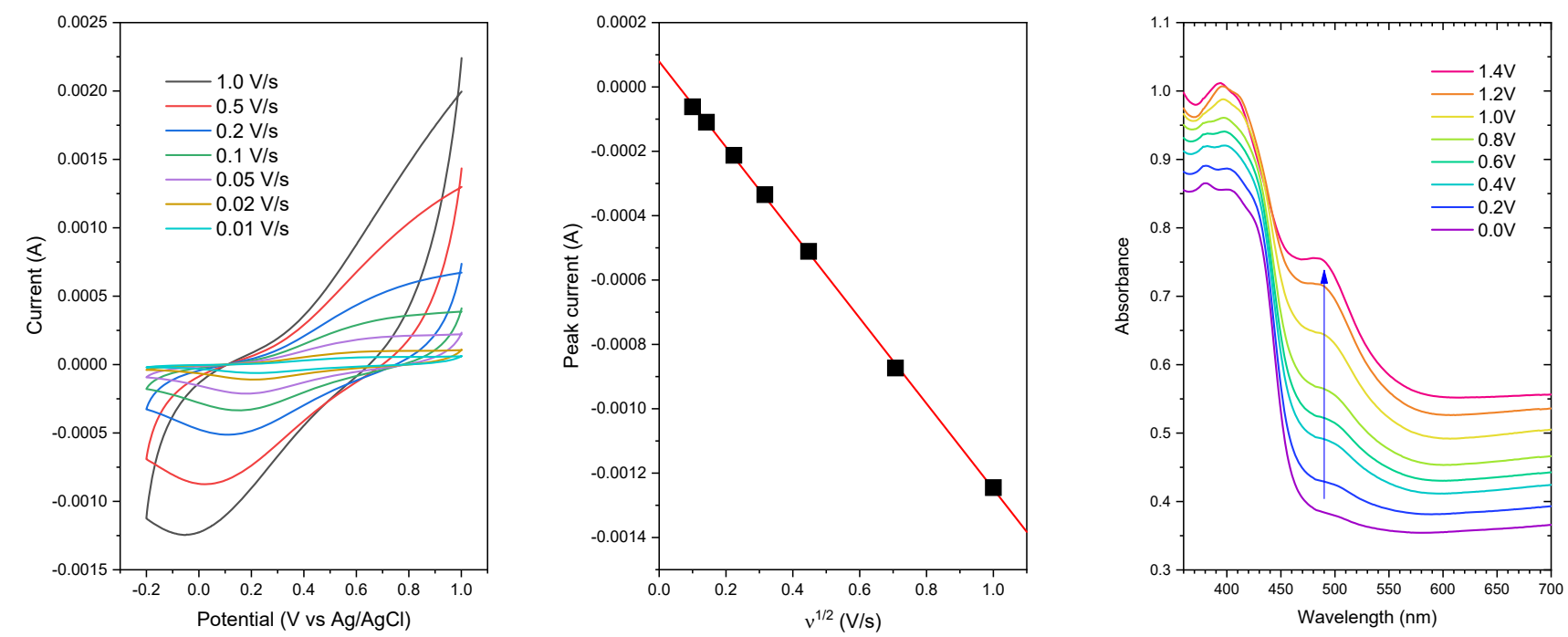

Figure 2: a) Cyclic voltammograms of a Mn-MOF-74 measured in 0.2M $\mathrm{LiClO}_{4}$ acetonitrile electrolyte solution at scan rates of $0.01,0.02,0.05,0.1,0.2,0.5$ and $1.0 \mathrm{~V} / \mathrm{s}$. b) The peak current vs square root of scan rate following a linear function. c) UV/Vis spectra of Mn-MOF74 biased at stepwise positive biasing up to $1.0 \mathrm{eV}$ in $0.2 \mathrm{M} \mathrm{LiClO}_{4}$ acetonitrile electrolyte solution. 


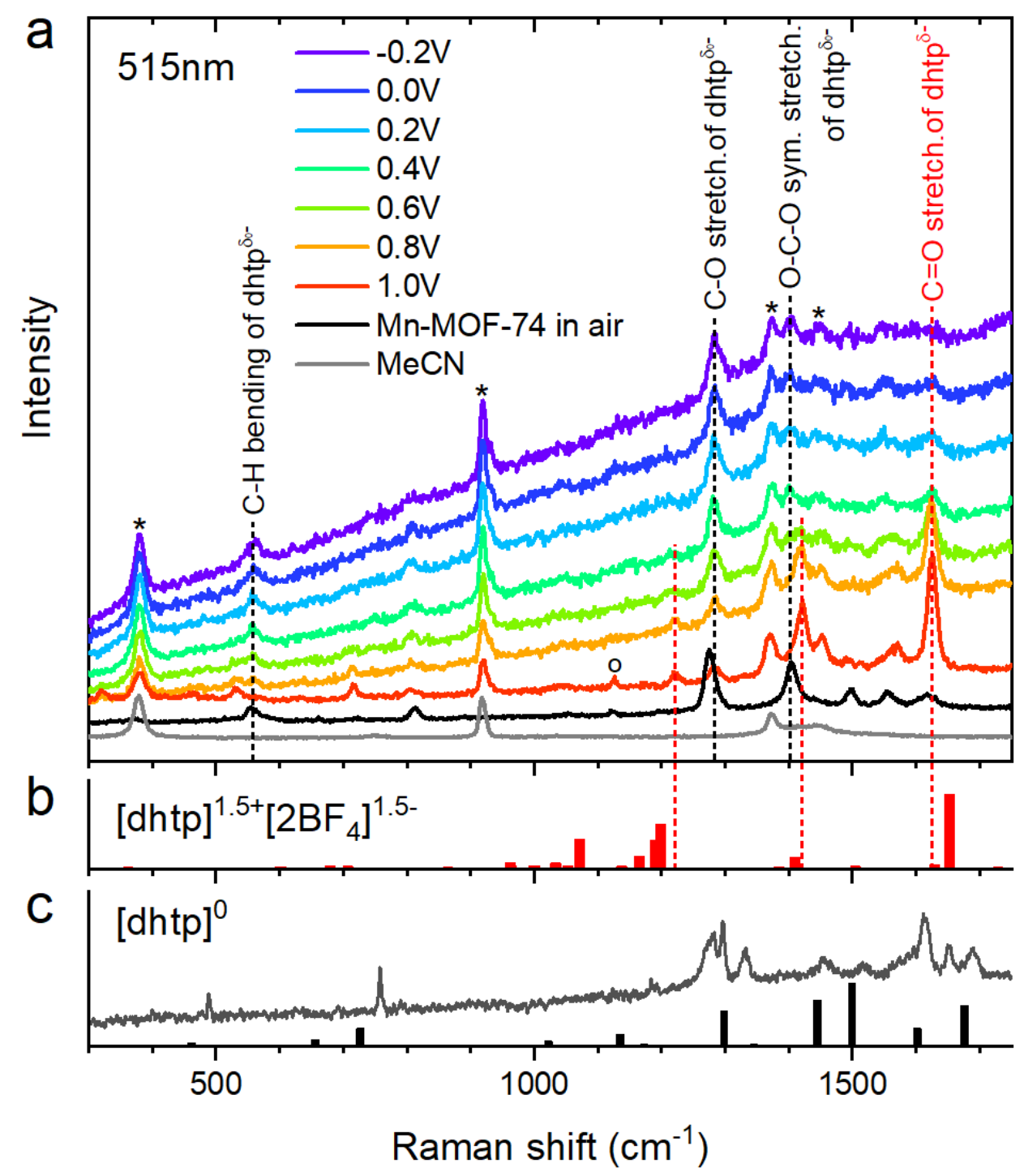

Figure 3: a. Raman spectra of Mn-MOF-74 biased at stepwise basing from $-0.2 \mathrm{~V}$ to $1.0 \mathrm{eV}$ in $0.2 \mathrm{M} \mathrm{LiClO}_{4}$ acetonitrile electrolyte solution, measured at a laser energy of $515 \mathrm{~nm}$. The asterisks indicates the peaks of Acetonitrile $(\mathrm{MeCN})$. The cirle indicates the peak of stray light. b. Theoretical Raman lines of $[\mathrm{dhtp}]^{1.46-}\left[2 \mathrm{BF}_{4}\right]^{1.46+}$ calculated based on the density functional perturbation theory. c. FT Raman spectrum of dhtp measured at a laser wavelength of $1064 \mathrm{~nm}$. The vertical bars are theoretical Raman lines of [dhtp] ${ }^{0}$. 

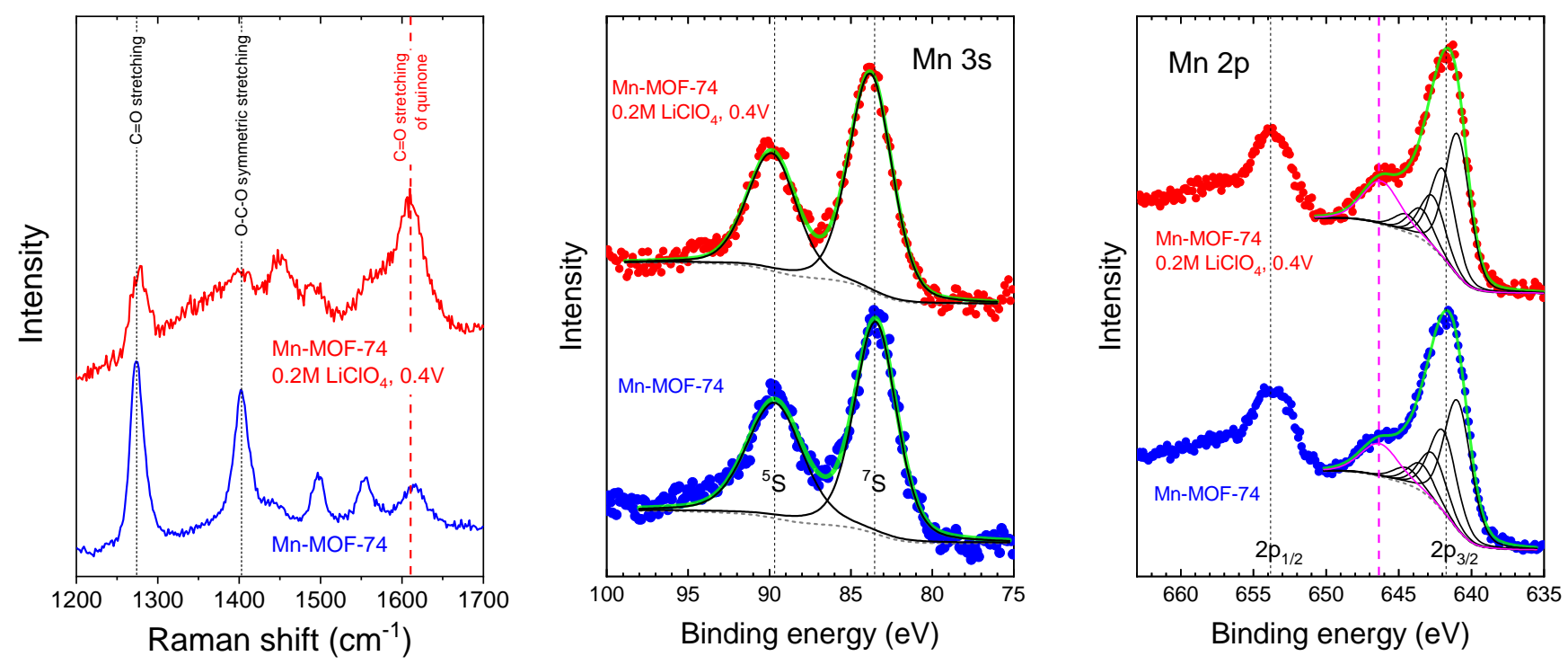

Figure 4: a) Raman (a) Mn 3s photoemission (b) and Mn 2p photoemission (c) spectra of doped and non-doped Mn-MOF-74. The overlapping spectral features were resolved into individual peaks using the damped non-linear least squares method after subtraction of Shirley background using Gaussian-Lorentzian line shape. 

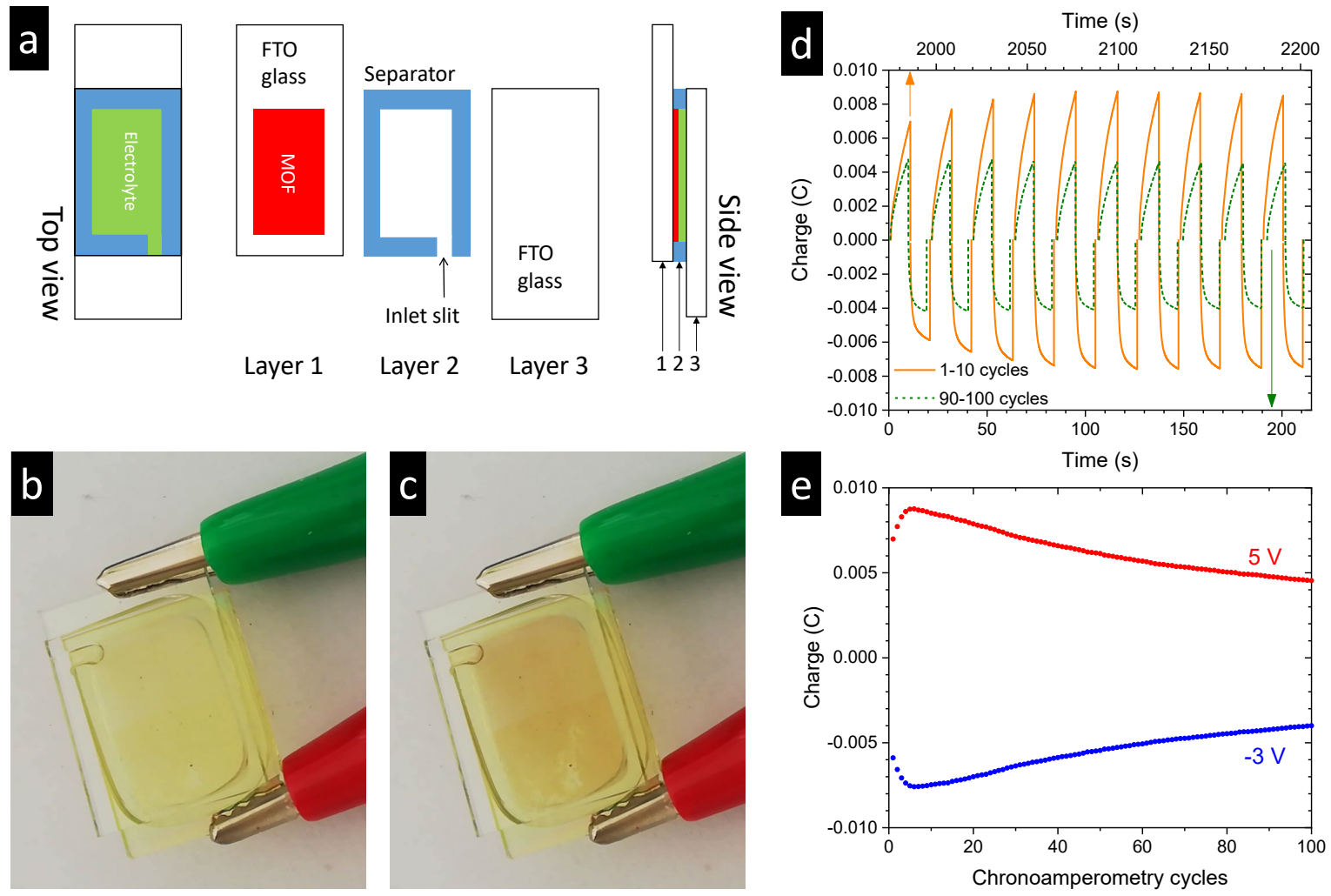

Figure 5: a) A diagram of the electrochromic device. Layer 1 on which a MOF film is deposited on, and layer 2 and 3 are glued together encapsulating electrolyte. b) Mn-MOF74-based electrochromic device encapsulating $0.2 \mathrm{M} \mathrm{LiClO}_{4}$ acetonitrile electrolyte solution biased at -3 V. c) Mn-MOF-74-based electrochromic device encapsulating $0.2 \mathrm{M} \mathrm{LiClO}_{4}$ acetonitrile electrolyte solution biased at $+5 \mathrm{~V}$. d) The inset shows the chronoamperogram upon bias switching between $-3 \mathrm{~V}$ and $5 \mathrm{~V}$ at $95-100$ cycles. The corresponding maximum charge accumulated in the device upon biasing at $-3 \mathrm{~V}$ and $5 \mathrm{~V}$ for 10 seconds. e) Cyclic voltammogram of the Mn-MOF-74-based electrochromic device at a scan rate of $0.1 \mathrm{~V} / \mathrm{s}$ in the bias range between $-3 \mathrm{~V}$ and $5 \mathrm{~V}$. 